\title{
EFFECT OF TWO DIFFERENT SURFACE TREATMENTS ON MICRO-SHEAR BOND STRENGTH OF CAD/CAM AND HEAT PRESSED GLASS CERAMICS
}

\author{
Shereen Kotb Salem *, Ahmed Ziada** and Khaled Aly Elbanna***
}

\begin{abstract}
Objective: The aim of this study was to evaluate the micro-shear bond strength between a dual cured resin cement and five different types of ceramics in composition and techniques of construction, using two different surface treatments.

Materials \& methods: A total of 50 ceramic disks were made and divided into 5 equal groups according to material type IPS Empress CAD, IPS e.max Press, IPS e.max CAD, Vita Suprinity and PM9 $(n=10)$. Each group was subdivided into 2 subgroups according to surface treatment they will be subjected to ( $n=5)$. Subgroup I: Bonding surfaces were etched with $5 \% \mathrm{HF}$ acid, then coated with Monobond S. Subgroup II: Monobond Etch \& Prime was applied on the bonding surfaces. For standardization of bonding surface area, 5 plastic tubings distributed on the bonding surface of each disk were used to place the dual resin cement. The samples were then subjected to micro-shear bond strength.
\end{abstract}

Results: Results showed insignificant difference in micro-shear mean values between the IPS Empress CAD subgroups while significant differences were found between the other subgroups within the same group. Monobond Etch \& Prime surface treatment resulted in higher micro-shear bond strength compared with HF surface treated subgroups for all the tested materials except for the PM9 and IPS e.max CAD.

Conclusions: It was found that ceramic microstructure as well as the fabrication technique affects the selection surface treatment type. Also, Monobod Etch and Prime as a single step surface treatment produced clinically accepted and comparable results to that of HF treatment.

KEY WORDS: Micro-shear, bond strength, Hydrofluoric acid, Monobond Etch \& Prime, Surface treatment, Glass ceramic, Pressable, CAD/CAM

*Lecturer of Fixed Prosthodontics. Faculty of Dentistry, October 6 University

**Lecturer of Fixed Prosthodontics, Faculty of Dentistry, Benisuef University.

***Assistant Professor of Fixed Prosthodontics. Faculty of Dentistry, Umm Alqura University 


\section{INTRODUCTION}

Growing interest in all ceramic esthetic dental restorations due to their superior colour and translucency, led to the continuous improvement and innovation in the composition of dental ceramics, manufacturing techniques and cementing systems, seeking improvement of materials' mechanical strength, better bonding capacity, as well as enamellike appearance. ${ }^{(1-5)}$ To improve the mechanical properties, ceramics reinforced with leucite, lithium disilicate and zirconia were introduced.

Ceramics are brittle materials that require proper bonding to tooth structure using cement. ${ }^{(6,7)}$ Their clinical success as dental restorations is determined by bond durability and strength of the resin cement to the ceramic material and to tooth structure. ${ }^{(8-11)}$ Stewart et al. $\mathbf{( 2 0 0 2}^{(12)}$ and Fabianelli et al (2010), ${ }^{(13)}$ reported that during bonding ceramic to tooth structure, the enamel or dentin/cement interfaces as well as the ceramic/cement interface should be optimized as the weaker one will determine the restoration's final bond strength. Failure of the adhesive seal in any of these interfaces, results in microleakage jeopardizing the longevity and clinical performance of the restorations, causing stains, recurrent caries, sensitivity adverse pulp response and ends with restoration debonding.(14) Also proper adhesive bonding increases the ceramic fracture resistance by up to $69 \%{ }^{(15)}$ by allowing load distribution through the bonding interface ${ }^{(16)}$ improving resistance and preventing crack propagation. Thus, increases the clinical longevity. ${ }^{(17)}$ Many researchers, ${ }^{(18-23)}$ concluded that cementation procedures, ceramic materials type, surface conditioning material and bonding techniques as well as cementing agents are very important to determine the success of all ceramic restorations.

Several researches ${ }^{(24-30)}$ studied different ceramic surface treatments used to obtain an optimum ceramic cement interface. Tooth and ceramic surfaces can be treated with acidic solutions to provide greater bonding capacity to adhesive cementing systems. ${ }^{(4,8-11)}$

Regarding glass ceramic surface, they proposed conditioning with hydrofluoric acid (HF) etching to gain micromechanical retention followed by silanation for chemical bonding with the resin cement. ${ }^{(31)} \mathrm{HF}$ causes selective dissolution of the glass matrix creating micromechanical retention. Silanation helps for the chemical adhesion between the organic and inorganic substances, producing a strong and durable adhesive bond between the ceramic and the resin cement. ${ }^{(13,32)}$ Silane coupling agent bonds resin composites to silica-based ceramics. ${ }^{(30,33)}$ During composite resin curing, an addition polymerization reaction occurs between the methacrylate groups of the resin matrix and silane bifunctional groups. While bonding with ceramics occurs through a condensation reaction between the silanol $(\mathrm{Si}-\mathrm{OH})$ groups on the ceramic surface and those of the hydrolyzed silane molecules. ${ }^{(34)}$

$\mathrm{HF}$ as an etchant for dental ceramics has the risk of toxic accidents as it is highly corrosive and in high concentrations, it can be absorbed through the skin into blood and bone. ${ }^{(35-38)}$ Also, extended application of HF may have a weakening effect on the ceramic surface and deteriorate the mechanical properties due to formation of insoluble silica fluoride salts as by-products on the surface. ${ }^{(13,20}$ 36-40) Multiple etching agents are introduced for investigation as an alternate to HF. However, none resulted in comparable outcomes to the use of HF acid. ${ }^{(41,42)}$

Universal adhesives represent the latest generation of dental adhesives that failed to prove their manufacturers' claims that they can be used as bondng agents on ceramic surfaces after HF surface treatment. ${ }^{(43-45)}$ Recently, a novel single bottle selfetching ceramic primer, (Monobond ${ }^{\circledR}$ etch \& prime), (Ivoclar Vivadent; Schaan, Liechtenstein), was introduced. It has the advantage of easier handling, less technique sensitivity and less time consumption (Single step). It is based on an alcoholic-aqueous solution of ammonium polyfluoride and silane methacrylate. Ammonium polyfluoride causes the 
production of a rough etching pattern, while the thin uniform layer of silane promotes the chemical bond to the glass ceramic. ${ }^{(45)}$ It is claimed that the effect of Monobond Etch \& Prime is comparable to those of HF and silane treatment. ${ }^{(46-48)}$ However, very few in-vitro or in-vivo studies have been reported with limited data.

Many bond strength tests were used to evaluate bond strength yet, shear bond strength test has become an accepted evaluation method. Shear stresses are considered major stresses responsible for in-vivo restorative bonding failures. ${ }^{(49)}$

The aim of this study was to evaluate the micro-shear bond strength between a dual cured resin cement and two leucite-reinforced ceramics, two lithium disilicate reinforced ceramics and $\mathrm{Zr}$ reinforced lithium silicate using two different surface treatments \& two different techniques of construction.

The hypothesis is that the micro-shear bond strength will vary according to the different surface treatment used with different ceramic structures and technique of construction.

TABLE (1)

\section{MATERIALS AND METHODS}

\section{Materials}

The materials used in this study are listed in Table 1.

\section{Sample Classification}

A total of 50 disks $(10 \mathrm{~mm}$ diameter and $2 \mathrm{~mm}$ height) were made and divided into 5 equal groups according to material type $(\mathrm{n}=10)$ and each group was further subdivided into 2 subgroups according to surface treatment they will be subjected to $(n=5)$. The group distribution is shown in table 2 .

\section{METHODS}

\section{Discs Fabrication}

\section{A) Pressable ceramics}

The ceramic discs of groups II and V were manufactured as follows:

\begin{tabular}{|l|l|}
\hline \multicolumn{1}{|c|}{ Brand Name and Manufacturer } & \multicolumn{1}{c|}{ Description } \\
\hline IPS Empress CAD (IvoclarVivadent, Schaan, Lichtenstein) & CAD/CAM Leucite-based ceramic \\
\hline IPS e.max Press (Ivoclar Vivadent, Schaan, Lichtenstein) & Pressable Lithium disilicate based ceramic \\
\hline IPS e.max CAD (Ivoclar Vivadent, Schaan, Lichtenstein) & CAD/CAM Lithium disilicate based ceramic- \\
\hline Vita Suprinity (Vita Zahnfabrik, Badsackingen, Germany) & $\begin{array}{l}\text { CAD/CAM Lithium silicate based ceramic }+8-12 \% \\
\text { zirconia }\end{array}$ \\
\hline PM9 (Vita Zahnfabrik, Badsackingen, Germany) & Presssable leucite reinforce ceramic \\
\hline IPS ceramic etching gel (Ivoclar Vivadent, Schaan, Lichtenstein) & $5 \%$ hydrofluoric acid Ceramic etchant \\
\hline Monobond S (IvoclarVivadent, Schaan, Lichtenstein) & Silane coupling agent \\
\hline Monobond Etch \& Prime (IvoclarVivadent, Schaan, Lichtenstein) & Ammonium Polyfluride + silane coupling agent \\
\hline Variolink Esthetic DC (Ivoclar Vivadent, Schaan, Lichtenstein) & Dual-cure adhesive resin cement \\
\hline
\end{tabular}


TABLE (2)

\begin{tabular}{|c|c|c|c|c|c|}
\hline & Group I & Group II & Group III & Group IV & Group V \\
\hline $\begin{array}{l}\text { Subgroup } 1 \\
\text { HF+ Silane }\end{array}$ & $\begin{array}{c}\text { IPS Empress } \\
\text { CAD + } \\
\mathrm{HF}+\text { silane }\end{array}$ & $\begin{array}{l}\text { IPS e.max Press } \\
+\mathrm{HF}+\text { silane }\end{array}$ & $\begin{array}{l}\text { IPS e.max CAD } \\
+\mathrm{HF}+\text { silane }\end{array}$ & $\begin{array}{c}\text { Suprinity } \\
(\text { pressable })+\mathrm{HF}+ \\
\text { silane }\end{array}$ & $\begin{array}{c}\text { PM9 + } \\
\mathrm{HF}+\text { silane }\end{array}$ \\
\hline $\begin{array}{c}\text { Subgroup } 2 \\
\text { Monobond Etch } \\
\text { \&Prime }\end{array}$ & $\begin{array}{l}\text { IPS Empress } \\
\text { CAD } \\
+ \text { Etch \& Prime }\end{array}$ & $\begin{array}{l}\text { IPS e.max Press } \\
+ \text { Etch \& Prime }\end{array}$ & $\begin{array}{l}\text { IPS e.max CAD } \\
+ \text { Etch \& Prime }\end{array}$ & $\begin{array}{c}\text { Suprinity } \\
+ \text { Etch \& Prime }\end{array}$ & $\begin{array}{c}\text { PM9 } \\
+ \text { Etch \& Prime }\end{array}$ \\
\hline
\end{tabular}

\section{Wax Pattern Preparation:}

Twenty wax patterns were made from dipping wax (BEGO, Germany). For standardization of wax dimensions, a special metal ring was fabricated with $10 \mathrm{~mm}$ diameter and $2 \mathrm{~mm}$ height. The wax was poured in the ring until the ring was filled completely with the wax and left to cool down to room temperature. A glass slab was put on the top of the metal ring with the poured wax to ensure flatness of the wax pattern.

\section{Investing Procedure:}

The wax patterns were sprued and invested using IPS PressVest Premium (Ivoclar Vivadent, Schaan, Lichtenstein) and Vita PM (Vita Zahnfabrik, Bad Sackingen, Germany) for groups II and V respectively.

\section{Wax Elimination:}

After the investment materials set, the rings were placed in Midtherm furnace (BEGO, Germany) to eliminate the wax from the ring.

\section{Heat Pressing}

After the wax elimination, IPS e.max Press (Ivoclar Vivadent, Schaan, Lichtenstein) and PM9 ingots (Vita Zahnfabrik, Bad Sackingen, Germany) were put in the rings then placed in Programat EP3010 (Ivoclar Vivadent, Schaan,Lichtenstein) and Vacumat 6000 M (Vita Zahnfabrik, Bad Sackingen, Germany) for groups II and V respectively.
The discs were divested and the sprue was cut. Alumina particles $(110 \mu)$ were used for sandblasting of the surfaces using Protempomatic (BEGO, Germany).

\section{B) CAD/CAM ceramics:}

Three groups of ceramic discs were constructed from leucite glass ceramic (IPS Empress CAD, Ivoclar Vivadent, Schaan, Liechtenstein), lithium disilicate glass- ceramic (IPS e.max CAD, Ivoclar Vivadent, Schaan, Liechtenstein) and zirconia-reinforced lithium silicate glass ceramic (ZLS, Vita Suprinity, Vita Zahnfabrik, Bad Sackingen, Germany) using CAD/ CAM system (Cerec inLab, Sirona, Germany). Each group consisted of ten discs of 10 $\mathrm{mm}$ diameter and $2 \mathrm{~mm}$ thickness. In order to standardize the disc dimensions, a machine-made metal mold with a cavity of $10 \mathrm{~mm}$ diameter and $2 \mathrm{~mm}$ thickness was used.

The upper surface of the metal mold including the cavity with the standardized dimensions was sprayed with scan spray (Cerec Optispray, Sirona) for scanning in the inLab scanner (inEos, Sirona, Germany).The design of the disc shaped samples was chosen using the CerecInLab software. IPS Empress CAD, IPS e.max CAD and Vita Suprinity blocks were milled using the CAD/CAM milling machine (Cerec inLab MC XL milling machine, Sirona, Germany) to obtain ten discs for each ceramic material.

IPS e.max CAD discs were fully crystallized in a furnace (Programat P500, Ivoclar Vivadent, Schaan, Liechtenstein) for thirty minutes at $850^{\circ} \mathrm{C}$ according 
to manufacturer instructions. Vita Suprinity disc samples were fully crystallized in the Programat furnace at $840^{\circ} \mathrm{C}$ according to manufacturer instructions.

Self-cure acrylic resin was mixed and poured in a custom-made housing and each disc was inserted in the acrylic resin before setting and then left to set in the housing. The function of the acrylic resin housing was to facilitate handling of the ceramic discs during surface treatment and testing.

\section{Surface treatment:}

Subgroup I: The bonding surfaces were etched with 5\% HF acid (IPS ceramic etching gel. Ivoclar Vivadent, Liechtenstein) according to the manufacturer recommendations (20 seconds for IPS e.max Press, IPS e.max CAD and Vita Suprinity while 60 seconds where recommended for etching of IPS Empress CAD and PM9), then rinsed, and dried for 10 seconds, then coated with Monobond S (Ivoclar Vivadent, Liechtenstein) using a microbrush and left to react for 60 seconds, and then dried.

Subgroup II: Monobond Etch \& Prime (Ivoclar Vivadent, Liechtenstein) was applied on the bonding surfaces and agitated by micro-brush for 20 seconds and left to react for another 40 seconds, then rinsed and dried for 10 seconds according to the manufacturer instructions.

\section{Cement application:}

For standardization of bonding surface area, plastic tubings with an inner diameter of $1 \mathrm{~mm}$ and $1 \mathrm{~mm}$ height were used to place the resin cement on the ceramic bonding surfaces. Five tubes were distributed on the bonding surface of each disk. (50-52) Variolink Esthetic DC adhesive resin cement was auto-mixed and applied through the tubes to the ceramic bonding area using the auto-mixing syringe, to the height of $1 \mathrm{~mm}$ and then light cured according to the manufacturer's instructions for 10 $\mathrm{s}$ from all directions using Optilux 400 system (3M ESPE, Seefeld, Germany). The tubes were then removed leaving a resin cement cylinder of $1 \mathrm{~mm}$ height and 1 diameter. The samples were then stored in saline for 24 hours before testing the micro-shear bond strength. ${ }^{(12)}$

\section{Micro-shear Bond Strength Testing:}

Micro-shear bond tests were performed using Bluehill Lite Software from Instron ${ }^{\circledR}$. Each disc with its own bonded micro-cylinders was mounted into central hole of especially designed sample holder Jakob's jig which in turn secured horizontally with tightening screws (Figure 1) to the lower fixed compartment of a materials testing machine (Model 3345; Instron Industrial Products, Norwood, MA, USA) with a load cell of $5 \mathrm{KN}$ and data were recorded using computer software (Instron ${ }^{\circledR}$ Bluehill Lite Software). An orthodontic wire (0.014" in diameter) was twisted in a form of a loop wrapped around the bonded micro-cylinder assembly at the ceramicresin interface and aligned with the loading axis of the testing machine upper movable compartment. A shearing load with tensile mode of force was applied via materials testing machine at a crosshead speed of $0.5 \mathrm{~mm} / \mathrm{min}$. The load required to de-bonding was recorded in Newton.

\section{Micro-Shear bond strength calculation;}

The load at failure was divided by bonding area to express the bond strength in $\mathrm{MPa}: \boldsymbol{\tau}=\mathbf{P} / \boldsymbol{\pi \mathbf { r } ^ { 2 }}$

Where; $\boldsymbol{\tau}=\mu$-shear bond strength (in MPa), $\mathbf{P}$ $=$ load at failure (in $\mathrm{N}$ ), $\boldsymbol{\pi}=3.14$ and $\mathbf{r}=$ radius of micro-cylinder (in $\mathrm{mm}$ )

\section{RESULTS}

Means and standard deviations of micro- shear bond strength values for the tested groups and subgroups are presented in Table $3 \&$ Figure (2). Two way ANOVA test followed by Fisher's LSD Method were used to determine the significant differences between the tested groups and subgroups $(\mathrm{p}<0.05)$. Results showed insignificant difference in microshear mean values between the IPS Empress CAD subgroups while significant differences were found 


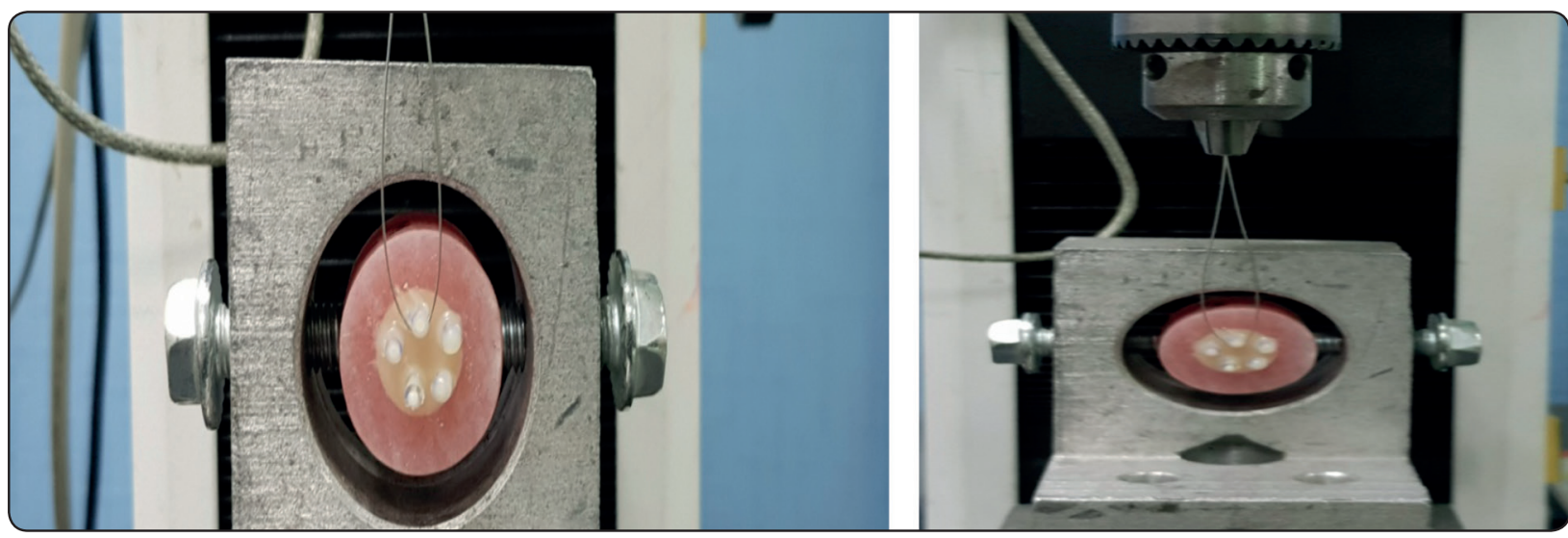

Fig. (1): A mounted disc secured horizontally with tightening screws to the lower fixed compartment of a materials testing Instron machine.

between the other subgroups within the same group (Groups II, III, IV and V). Monobond Etch \& Prime surface treatment resulted in higher micro-shear bond strength compared with HF surface treated subgroups for all the tested materials except for the PM9 and IPS e.max CAD.

The highest micro-shear bond strength was recorded with the HF treated IPS e.max CAD samples (31.9461 MPa), while the lowest was recorded for HF treated IPS e.max press samples (19.04301 MPa). However, the highest Monobond Etch \& Prime results were recorded by the Vita Suprinty samples (31.55024 MPa) while the lowest were recorded by the PM9 samples (19.51466 MPa).

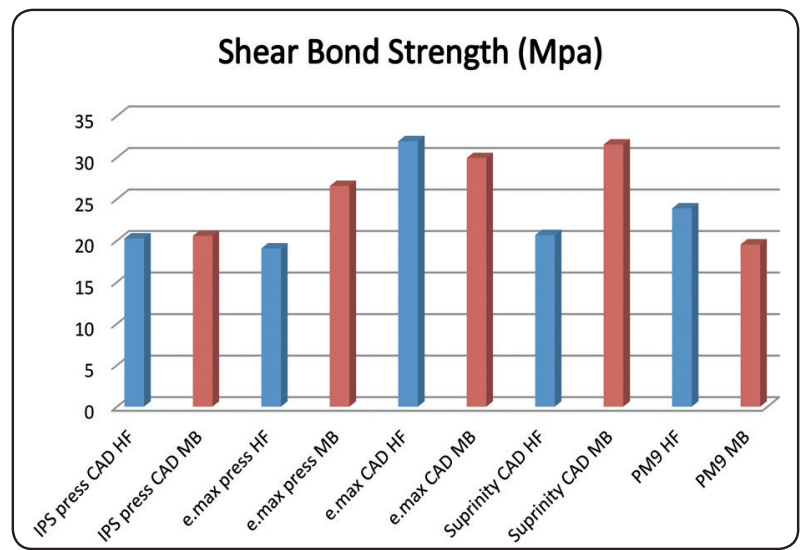

Fig. (2) Comparison between the tested subgroups

TABLE (3) Means \& Standard deviations of shear bond strength of the tested groups in MPa.

\begin{tabular}{|c|c|c|c|c|c|c|}
\hline & Group I & Group II & Group III & Group IV & Group V & \\
\hline & $\begin{array}{c}\text { IPS Empress } \\
\text { CAD }\end{array}$ & $\begin{array}{l}\text { IPS e.max } \\
\text { Press }\end{array}$ & $\begin{array}{l}\text { IPS e.max } \\
\text { CAD }\end{array}$ & Suprinity CAD & PM9 & Critical Values \\
\hline HF & $\begin{array}{l}20.23688^{a} \\
(6.11718)\end{array}$ & $\begin{array}{c}19.04301 \mathrm{a} \\
(2.00200)\end{array}$ & $\begin{array}{l}31.9461 b \\
(6.32016)\end{array}$ & $\begin{array}{l}20.65168^{\mathrm{a}} \\
(2.59157)\end{array}$ & $\begin{array}{l}23.86693 \mathrm{c} \\
(5.98495)\end{array}$ & $\begin{array}{c}\text { Between columns } \\
\text { (Ceramics): } \\
2.48773\end{array}$ \\
\hline MB & $\begin{array}{l}20.55482^{\mathrm{a}} \\
(4.50934)\end{array}$ & $\begin{array}{l}26.55788^{d} \\
(5.49645)\end{array}$ & $\begin{array}{l}29.92683^{\mathrm{e}} \\
(5.22272)\end{array}$ & $\begin{array}{l}31.55024^{\mathrm{b}} \\
(3.27378)\end{array}$ & $\begin{array}{l}19.51466^{\mathrm{a}} \\
(2.46860)\end{array}$ & $\begin{array}{c}\text { Between } \\
\text { Rows (Surface } \\
\text { treatments): } \\
0.99509\end{array}$ \\
\hline
\end{tabular}




\section{DISCUSSION}

Five glass ceramic materials were treated with two different surface treatments and the shear bond strength to a dual cured resin cement was evaluated.

The ceramic materials were selected to represent most of the available glass ceramic microstructures as well as different manufacturing techniques to investigate whether the microstructure and manufacturing technique affect the choice of surface treatment in regards to shear bond strength or not.

The lithium disilicate reinforced ceramic was represented by IPS e.max Press and IPS e.max CAD. Leucite reinforced ceramic was represented by PM9 and IPS Empress CAD. Also, the zirconia reinforced lithium silicate was represented by the Vita Suprinity CAD/CAM ceramic.

As the bond strength and durability at the ceramic-resin interface depend on surface treatment selection that is suitable for the ceramic microstructure, ${ }^{(27,29,53)}$ acid etching using $5 \%$ HF etching was proposed by the manufacturers for surface treatment of the ceramics used in this study. Time of ceramics' exposure to HF was set according to the manufacturer recommendations as increasing the acid concentration or extended HF application may deteriorate the glass ceramics bonding and mechanical properties. ${ }^{(18)}$ This mechanical weakness was attributed to the production of insoluble silica fluoride salts as by-products on the ceramic surface. These by-products were accused of weakening the bond strength with resins. ${ }^{(13,39,40)}$ Monobond-S primer is recommended by the manufacturer to be used with variolink resin cement.

As multi-step surface treatment technique is technique sensitive, time consuming consequently, it may compromise bonding effectiveness. The search for an alternative seemed to be mandatory. That's why Monobond Etch \& Prime (Ivoclar, Vivadent) glass primer was used in this study due to its offering a safe single step procedure for glass ceramic conditioning.
Conventional ceramics rich in the glass phase form high bond strength to resin cements. ${ }^{(27,29,53)}$ Variolink Esthetic DC adhesive resin cement was used, as dual-cured resin cements have the advantages of adequate polymerization in light inaccessible areas as well as controlled working time. ${ }^{(54)}$ Dual-cured resin cements polymerization as reported by many researchers should be initially light activated to reach their optimum mechanical properties as photo-activation increases the degree of conversion. ${ }^{(55-60)}$

In this study bond strength evaluation of the tested ceramics to the dual cured cement was done using micro - shear bond strength test as shear stresses are considered the major stresses responsible for invivo bond failure. ${ }^{(49)}$ The used micro-shear testing methodology was introduced by Shimada et al (2002). ${ }^{(40)}$ This type of mechanical test solves the problem of propagation of tensile stresses at a large bonded interface. Also, it possesses the advantage of taking several readings from one sample, as if we multiplied the number of samples. ${ }^{(61)}$

For standardization of bonding surface area, plastic tubings with inner diameter of $1 \mathrm{~mm}$ and 1 $\mathrm{mm}$ height were used to place the resin cement on the ceramic bonding surfaces.

Eventually, all the tested sub-groups showed micro-shear bond strength mean value above the minimal clinically accepted micro-shear bond strength value for bonded restorations; 10-13 MPa. ${ }^{(26)}$

Among the leucite reinforced ceramics: the IPS Empress CAD and the PM9 sub-groups, the only significant difference was found with the PM9 subgroup treated with HF showing less micro-shear bond strength.

These results can be attributed to the difference in leucite concentration between IPS Empress CAD and PM9, size, orientation of crystals and microstructure which affect etching times and consequently the bond strength. As the diameter of leucite crystals in IPS Empress CAD 1-5 $\mu$. They are evenly and densely distributed with crystals 
$35-40 \%$ by volume. According to the manufacturer's scientific documentation, etching dissolves leucite crystals more quickly than the glass matrix. ${ }^{(62)}$

Concerning the lithium disilcate ceramics represented in this study by the IPS e.max Press and IPS e.max CAD, the significant difference found between the 4 subgroups came on the contrary to Alrahlah et al (2017), ${ }^{(63)}$ who recorded comparable shear bond strength results between resin cement and lithium disilicate samples treated with $\mathrm{HF}+$ silane and Monobond Etch \& Prime. He attributed these results to that Monobond Etch \& Prime contains trimethoxypropyl methacrylate for silanization and polyfluoride for etching. SEM micrographs showed that the HF etched IPS e.max Press samples presented more prominent crystals with higher retentive shadow irregularities compared to the Monobond samples that revealed less prominent, and fewer crystals on the primed surfaces, in addition to no shadow retentive irregularities.

As the manufacturer claims, Monobond Etch \& Prime achieves similar bond strength as the combination of HF etching + silane application. Monobond in-spite of producing the less pronounced rough etching pattern, a highly effective chemical bond is formed due to the ammonium polyfluoride ions induction of reactive silanol groups formation that become no longer stabilized after the ceramic rinsing and polyfluoride removal. It was reported that the bond between silica and fluoride is extremely strong. Therefore, the effective bonding may be attributed to the chemical affinity between silica in lithium disilicate and ammonium trifluoride in Monobond. ${ }^{(45,64)}$ These finding were supported by many researchers, ${ }^{(65,66)}$ who recorded morphological observations of silica glass matrix in lithium disilicate. The displaced glass matrix due to the polyfluoride component of Monobond, becomes incorporated into the adhesive bond with the resin cement. This creates a complex layer of fluoride, silica, silane molecules, and polymerizing monomers and polymers, which are highly critical to the lithium disilicate bonding and clinical success. ${ }^{(67)}$
This may explain the significant difference between the IPS e.max Press subgroups, with the Monobond sub-group showing higher results.

Prado et al (2018), ${ }^{(68)}$ tested the effect of two surface treatments on machined slices of lithium disilicate ceramic (IPS e.max CAD) and feldspathic ceramic (Vita Mark II). They used 5\% HF followed by silane application and Monobond Etch \& Prime. Although HF + Silane resulted in higher mean microshear bond strength than Monobond Etch \& Prime for both ceramics as in our study yet, Monobond Etch \& Prime had stable bonding after aging. They attributed the higher bond strength of the groups treated with $\mathrm{HF}$ to the pronounced topographical alterations found in SEM micrographs of the surface of HF samples compared to Monobond Etch $\&$ Prime groups for both ceramics. HF acid etching revealed the presence of elongated crystals and surface irregularities due to the dissolution of the glassy matrix of lithium disilicate ceramic.

Results were in accordance with those found by El-Damanhoury and Gaintantzopoulou(2018), ${ }^{(69)}$ in which Monobond Etch \& Prime resulted in a smoother surface (ie, fewer irregularities for micromechanical interlocking), and lower bond strengths than HF + silane. The slight topographic alterations generated by Monobond Etch and Prime compared with HF corroborate the findings by Siqueira et al (2016). ${ }^{(47)}$

They explained these findings by the fact that Monobond Etch \& Prime contains $\leq 10 \%$ of tetrabutyl ammonium dihydrogen trifluoride which is less acidic than $\mathrm{HF}^{(18)}$ to obtain an irregular pattern on the ceramic surface and micromechanical retention. ${ }^{(47,69)}$

The significant difference between the IPS e.max Press and the IPS e.max CAD groups and the contrasting results having higher bond in the Monobond treated IPS e.max Press subgroup and the HF treated IPS e.max CAD subgroup may be attributed to the difference in microstructure of the IPS e.max Press with the main crystal phase, 70\% needle like crystals 3-6 $\mu$ m length lithium disilicate 
crystals $\mathrm{LiSi}_{2} \mathrm{O}_{5}$ embedded in glass matrix. While for IPS e.max CAD, the microstructure consists of $40 \%$ platelet shaped $0.2-1 \mu \mathrm{m}$ partially crystallized lithium metasilite $\mathrm{LiSiO}_{3}$ embedded in glassy matrix. The fully crystallized form contains approximately $70 \%$ fine grained lithium disilicate crystals. Also, the manufacturer recommended finishing of IPS e.max CAD restorations due to the fact that after $\mathrm{CAD} / \mathrm{CAM}$ ceramic milling the restoration shows detectable surface roughness which varies according the geometry of the milling tools and grain size. This enhances the effect of micromechanical bonding. This comes on the contrary to the unfinished press ceramic restorations that result from a viscous conversion of the press ingots resulting in a smooth surface during the hot pressing procedure. So they do not have such milling marks. However, the surface roughness of milled ceramic restorations can be reduced by surface finishing using diamonds.

On the other hand, Siqueira et al (2016) $)^{(47)}$ and Roman-Rodriguez et al (2018), ${ }^{(46)}$ found no statistically significant difference in micro-shear bond strength of a lithium disilicate ceramic etched with Monobond Etch \& Prime or HF

Since glass ceramics are more susceptible to acid etching than zirconia ceramics ${ }^{(70)}$ and as the silica content of zirconia reinforced lithium silicate ceramic (56-64\%) is similar to that of the other glass ceramics. Therefore, this material is considered susceptible to HF etching, unlike zirconia ceramics. ${ }^{71)}$

The significant difference between Vita Suprinity subgroups, with the MB subgroup(SCP) showing higher mean bond strength. Also, there was a significant difference between the micro-shear mean value of the Vita Suprinity and the IPS e.max CAD and IPS e.max Press subgroups in both types of surface treatments. This may be attributed to the homogeneous, fine crystalline micro- structure of $0.5 \mu \mathrm{m}$ of the Vita Suprinity compared to the IPS e.max CAD and IPS e.max Press. The smaller particle size for the same surface area may increase amount of pores, increasing the surface area available for bonding. ${ }^{(72)}$
These results were in agreement with DellaBona(2014), ${ }^{(73)}$ who concluded that ceramic microstructure affects the composite-ceramic adhesion zone and consequently the resin- ceramic bond strength. However, this was not in agreement with Janda et al(2003), ${ }^{(74)}$ Bottino et $\mathbf{a l}(\mathbf{2 0 0 5})^{(75)}$ and Amaral et al (2008), ${ }^{(76)}$ who claimed that addition $\mathrm{ZrO}_{2}$ resulted in more acid-resistant ceramics due to reduction in the glassy matrix and Si content.

The hypothesis was highly accepted as the micro-shear bond strength was affected by the surface treatment used, ceramic microstructure as well as the fabrication technique.

\section{CONCLUSIONS}

Therefore, within the limitations of this study it was found that ceramic microstructure as well as the fabrication technique affects the selection of surface treatment type. Also, Monobod Etch and Prime as a single step surface treatment produced clinically accepted and comparable results to that of HF treatment.

\section{REFERENCES}

1. Seiber C. In the light of nature. Quintessence Dent Technol 1993; 16:60-68.

2. Christensen JC. Porcelain-fused-to-metal vs. nonmetal crowns. J Am Dent Assoc 1999; 130:409-411.

3. Blatz MB, Sadan A, Kern M. Resin-ceramic bonding: a review of the literature. J Prosthet Dent. 2003 Mar; 89(3):268-274.

4. Radovic I, Monticelli F, Goracci C, Vulicevic ZR, Ferrari M. Self-adhesive resin cements: a literature review. J Adhes Dent 2008; 10:251-258.

5. Heintze SD, Cavalleri A, Zellweger G, Buchler A, Zappini G. Fracture frequency of all-ceramic during dynamic loading in a chewing simulator using different loading and luting protocols. Dent Mater 2008; 24:1352-1361.

6. Jensen ME, Sheth JJ, Tolliver D. Etched-porcelain resinbonded full-veneer crowns: in vitro fracture resistance. Compendium. 1989; 10 (6): 336-347. 
7. Burke FJ. The effect of variations in bonding procedure on fracture resistance of dentin-bonded all-ceramic crowns. Quintessence Int. 1995; 26: 293-300.

8. Peumans M, Van Meerbeek B, Lambrechts P, Vanherle G. Porcelain veneers: a review of the literature. J Dent. 2000 Mar;28 (3):163-177.

9. Della Bona A, Anusavice KJ, Shen C. Microtensile strength of composite bonded to hot-pressed ceramics. J Adhesive Dent. 2000; 2:305-313.

10. Della Bona A, Anusavice KJ, Mecholsky Junior JJ. Apparent interfacial fracture toughness of resin/ ceramic systems. J Dent Res. 2006; 85:1037-1041.

11. Ayad MF, Fahmy NZ, Rosenstiel SF. Effect of surface treatment on roughness and bond strength of a heat-pressed ceramic. J Prosthet Dent. 2008; 99:123-130.

12. Stewart GP, Jain P, Hodges J. Shear bond strength of resin cements to both ceramic and dentin. J Prosthet Dent. 2002 Sep;88(3):277-284

13. Fabianelli A, Pollington S, Papacchini F, Goracci C, Cantoro A, Ferrari M et al. The effect of different surface treatments on bond strength between leucite reinforced feldspathic ceramic and composite resin. J Dent. 2010 Jan; 38(1):39-43.

14. Turkun M, Cal E, Toman M, Toksavul S. Effects of dentin disinfectants on the shear bond strength of all ceramics to dentin. Oper Dent, 2005, 30 (4): 453-460.

15. Dietschi D, Spreafico R. Current clinical concepts for adhesive cementation of tooth-colored posterior restorations. Pract Periodontics Aesthet Dent. 1998; 10:47-54.

16. Attia A, Kern M. Influence of cyclic loading and luting agents on the fracture load of two all ceramic crown systems. J Prosthet Dent. 2004; 92:551-556.

17. Burke FJT, Wilson NHF, Watts DC. Fracture resistance of teeth restored with indirect composite resins: the effect of alternative luting procedures. Quintessence Int. 1994; 25: 269-275.

18. Hooshmand T, Parvizi S, Keshvad A. Effect of surface acid etching on the biaxial flexural strength of two hot-pressed glass ceramics. J Prosthodont.. 2008; 17: 415- 419.

19. Hooshmand T, Rostami G, Behroozibakhsh M, et al. Interfacial fracture toughness of different resin cements bonded to a lithium disilicate glass ceramic. J Dent. 2012; 40:139-145.
20. Marocho S, Ozcan M,Amaral R, et al.Effect of resin cement type on the microtensile bond strength to lithium disilicate ceramic and dentin using different test assemblies. J Adhes Dent. 2013; 15: 361-368.

21. Bitter K, Paris S, Hartwig C, et al. Shear bond strengths of different substrates bonded to lithium disilicate ceramics. Dent Mater J. 2006; 25:493-502.

22. de Carvalho RF, Martins ME, de Queiroz JR, Leite FP, Ozcan M. Influence of silane heat treatment on bond strength of resin cement to a feldspathic ceramic. Dent Mater J. 2011; 30: 392-397.

23. Della Bona A, Anusavice KJ. Microstructure, composition, and etching topography of dental ceramics. Int $\mathrm{J}$ Prosthodont. 2002; 15: 159-167.

24. Suliman AH, Swift EJ Jr, Perdigao J. Effects of surface treatment and bonding agents on bond strength of composite resin to porcelain. J Prosthet Dent. 1993; 70:118-120.

25. Ozden AN, Akaltan F, Can G. Effect of surface treatments of porcelain on the shear bond strength of applied dualcured cement. J Prosthet Dent. 1994; 72:85-88.

26. Thurmond JW, Barkmeier WW, Wilwerding TM. Effect of porcelain surface treatments on bond strengths of composite resin bonded to porcelain. J Prosthet Dent. 1994; 72: 355-359.

27. Roulet JF, Soderholm KJ, Longmate J. Effects of treatment and storage conditions on ceramic/composite bond strength. J Dent Res. 1995;74: 381-387.

28. Kupiec KA, Wuertz KM, Barkmeier WW, Wilwerding TM. Evaluation of porcelain surface treatments and agents for composite-to-porcelain repair. J Prosthet Dent. 1996; 76:119-124.

29. Kamada K, Yoshida K, Atsuta M. Effect of ceramic surface treatments on the bond of four resin luting agents to a ceramic material. J Prosthet Dent. 1998;79: 508-513.

30. Madani M, Chu FC, McDonald AV, Smales RJ. Effects of surface treatments on shear bond strengths between a resin cement and an alumina core. J Prosthet Dent. 2000;83: 644-647.

31. Hooshmand T, van Noort R, Keshvad A. Bond durability of the resin-bonded and silane treated ceramic surface. Dent Mater. 2002; 18:179-188.

32. Ozcan M, Valandro LF, Amaral R, Leite F, Bottino MA. Bond strength durability of a resin composite on a reinforced ceramic using various repair systems. Dent Mater. 2009 Dec;25(12):1477-1483. 
33. 35 Hayakawa T, Horie K, Aida M, et al. The influence of surface conditions and silane agents on the bond of resin to dental porcelain. Dent Mater. 1992; 8:238-240.

34. Ozcan M, Vallittu PK. Effect of surface conditioning methods on the bond strength of luting cement to ceramics. Dent Mater. 2003; 19:725-731.

35. Meldrum M. Toxicology of hydrogen fluoride in relation to major accident hazards. Regul Toxicol Pharmacol. 1999;30: 110-116.

36. Addison O, Fleming GJP. The influence of cement lute, thermocycling and surface preparation on the strength of a porcelain laminate veneering material. Dent Mater. 2004;20: 286-292.

37. Addison O, Marquis PM, Fleming GJP. The impact of hydrofluoric acid surface treatments on the performance of a porcelain laminate restorative material. Dent Mater. 2007;23: 461-468.

38. Saygili G, Sahmali S. Effect of ceramic surface treatment on the shear bond strengths of two resin luting agents to all-ceramic materials. J Oral Rehabil. 2003;30: 758-764..

39. Monticelli F, Toledano M, Osorio R, et al. Effect of temperature on the silane coupling agents when bonding core resin to quartz fiber posts. Dent Mater. 2006; 22: 1024-1028.

40. Shimada Y, Yamaguchi S, Tagami J. Micro-shear bond strength of dual-cured resin cement to glass ceramics. Dent Mater. 2002; 18:380-388.

41. Klosa K, Boesch I, Kem M. Long-term bond of glass ceramic and resin cement: evaluation of titanium tetrafluoride as an alternative etching agent for lithium disilicate ceramics. J Adhes Dent. 2013; 15: 377-383.

42. Kukiattrakoon B, Thammasitboon K. Optimal acidulated phosphate fluoride gel etching time for surface treatment of feldspathic porcelain: on shear bond strength to resin composite. Eur J Dent. 2012; 6: 63-69.

43. de Goes MF, Shinohara MS, Freitas MS. Performance of a new one-step multi-mode adhesive on etched vs nonetched enamel on bond strength and interfacial morphology. J Adhes Dent. 2014 Jun;16(3):243-50.

44. Muñoz MA, Sezinando A, Luque-Martinez I, et al. Influence of a hydrophobic resin coating on the bonding efficacy of three universal adhesives. J Dent. 2014;42: 595-602.

45. Scientific documentation of Monobond etch \& prime. Available from: http://www.ivoclarvivadent. us/en-us/p/ dental-professional/bonding-agents-luting-composites-torestorations/monobondetch- prime
46. Roman-Rodriguez JL, Perez-Barquero JA, GonzalezAngulo E, Fons-Font A, Bustos-Salvador JL. Bonding to silicate ceramics: Conventional technique compared with a simplified technique. J Clin Exp Dent. 2017;9: e384-e386.

47. Siqueira FS, Alessi RS, Cardenas AF, Kose C, Souza Pinto $\mathrm{SC}$, Bandeca MC, Loguercio AD, Gomes JC. New singlebottle ceramic primer: 6-month case report and laboratory performance. J Contemp Dent Pract. 2016;17: 1033-1039.

48. Wille S, Lehmann F, Kern M. Durability of resin bonding to lithium disilicate and zirconia ceramic using a self-etching primer. J Adhes Dent. 2017; 19: 491-496.

49. Watanabe LG, Marshall GW, Marshall SJ. Variables influence of shear bond strength testing to dentin. In: Tagami J, Toledano M, Prati C, editors. Advanced adhesive dentistry, 3rd international Kuraray Symposium. Kuraray Co. Ltd, Cirimido, Italy; 1999; 75-90.

50. Lee Y, Kim JH, Woo JS, Yi YA, Hwang JY, Seo DG. Analysis of self-adhesive resin cement microshear bond strength on leucite-reinforced glass-ceramic with/without pure silane primer or universal adhesive surface treatment. Biomed Res Int. 2015 Oct; 2015:361893.

51. McDonough W G, Antonucci J M, He J, Shimada Y, Chiang M Y M, Schumacher G E, Schultheisz C R. A microshear test to measure bond strengths of dentin-polymer interfaces. Biomater. 2002; 23: 3603-3608.

52. Chai Y, Lin h, Zheng G, Zhang X, Niu G, Du Q. Evaluation of the micro-shear bond strength of four adhesive systems to dentin with and without adhesive area limitation. Bio Med Mater Eng. 2005; 26:563-572.

53. Lacy AM, Laluz J, Watanabe LG, Dellinges M. Effect of porcelain surface treatment on the bond to composite. $\mathrm{J}$ Prosthet Dent. 1988; 760(3):288-291.

54. Lee IB, Um CM. Thermal analysis on the cure speed of dual cured resin cements under porcelain inlays. J Oral Rehab. 2001; 28(2):186-197.

55. Peutzfeldt A. Dual-cure resin cements: in vitro wear and effect of quantity of remaining double bonds, filler volume, and light curing. Acta Odont Scand 1995; 53(1):29-34.

56. Caughman WF, Chan DC, Rueggeberg FA. Curing potential of dual polymerizable resin cements in simulated clinical situations. J Prosthet Dent. 2001; 86(1):101-106.

57. El-Mowafy OM, Rubo MH, El-Badrawy WA. Hardening of new resin cements cured through a ceramic inlay. Oper Dent. 1999; 24(1):38-44. 
58. Hofmann N, Papsthart G, Hugo B, Klaiber B. Comparison of photo-activation versus chemical or dual-curing of resin-based luting cements regarding flexural strength, modulus of elasticity and surface hardness. J Oral Rehabil. 2001; 28(11):1022-1028.

59. Santos GC Jr, El-Mowafy O, Rubo JH, Santos MJ. Hardening of dual-cure resin cements and a resin composite restorative with QTH and LED curing units. J Can Dent Assoc. 2004; 70(5):323-328.

60. Rueggeberg FA, Caughman WF. The influence of light exposure on polymerization of dual-cure resin cements. Oper Dent. 1993; 18(2):48-55.

61. Sano H, Shono T, Sonoda H, Takatsu T, Ciucchi B, Carvalho RM. Relation between surface area for adhesion and tensile bond strength - evaluation of a microtensile bond test. Dent Mater. 1994 Jul;10(4):236-240.

62. Scientific Documentation IPS Empress CAD Ivoclar Vivadent AG. Available from: http://www.ivoclarvivadent.

63. Alrahlah A, Awad M M, Vohra F, Al-Mudahi A, Al jeaidi Z A, Elsharawy M. Effect of self etching ceramic primer and universal adhesive on bond strength of lithium disilicate ceramic. J Adhes Sci Technol. 2017; 31(23): 2611-2619.

64. Kolasinski KW. Etching of silicon in fluoride solutions. Surf Sci. 2009; 603:1904-1911.

65. Guarda GB, Correr AB, Gonçalves LS, et al. Effects of surface treatments, thermocycling, and cyclic loading on the bond strength of a resin cement bonded to a lithium disilicate glass ceramic. Oper Dent. 2013; 38:208-217.

66. Aboushelib MN, Sleem D. Microtensile bond strength of lithium disilicate ceramics to resin adhesives. J Adhes Dent. 2014; 16:547-52.

67. Xiaoping L, Dongfeng R, Silikas N. Effect of etching time and resin bond on the flexural strength of IPS e.max Press glass ceramic. Dent Mater. 2014;30: e330-e336.
68. Prado M, Prochnow C, Marchionatti A M E, Baldissara P / Luiz Felipe Valandro L F, Wandscher V F. Ceramic Surface Treatment with a Single-component Primer: Resin Adhesion to Glass Ceramics. J Adhes Dent. 2018; 20: 99-105.

69. El-Damanhoury HM, Gaintantzopoulou MD. Self-etching ceramic primer versus hydrofluoric acid etching: Etching efficacy and bonding performance. J Prosthodont Res. 2018; 62:75-83.

70. Lee H, Han G, Chan J, Son H. Bonding of the silane containing multi-mode universal adhesive for lithium disilicate ceramics. Restor Dent Endod. 2017; 42, 95-104.

71. Sato T, Anami L, Melo R, Valandro L, Bottino M. Effects of surface treatments on the bond strength between resin cement and a new zirconia-reinforced lithium silicate ceramic. Oper Dent. 2016; 41, 284-292.

72. VITA Zahnfabrik. Vita Suprinity Technical and scientific documentation. 2014. Available from: https://www.vitazahnfabrik.com.

73. Della-Bona A. Characterizing ceramics and the interfacial adhesion to resin: II- The relationship of surface treatment, bond strength, interfacial toughness and fractography. J Appl Oral Sci. 2005;13(2):101-109.

74. Janda R, Roulet J F, Wulf M, Tiller H J. A new adhesive technology for all-ceramics. Dent Mater. 2003;19(6): $567-573$.

75. Amaral R, Ozcan M, Valandro L F, Balducci I, Bottino M A. Effect of conditioning methods on the microtensile bond strength of phosphate monomer-based cement on zirconia ceramic in dry and aged conditions. J Biomed Mater Res B Appl Biomater. 2008; 85(1):1-9.

76. Bottino M A, Valandro L F, Scotti R, Buso L. Effect of surface treatments on the resin bond to zirconium-based ceramic. Int J Prosthodont. 2005;18(1):60-65. 